\section{MOVILIDAD, UTOPÍAS Y LUGARES HÍBRIDOS EN LOS ANDES DEL SUR DE CHILE ${ }^{1}$}

Viviana Huiliñir-Curío² y Hugo Marcelo Zunino³

\section{MOBILITY, UTOPIAS AND HYBRID PLACES IN THE SOUTHERN CHILEAN ANDES ${ }^{1}$}

Viviana Huiliñir-Curío $\&$ Hugo Marcelo Zunino ${ }^{3}$

\section{Resumen}

En la cordillera de Los Andes del sur de Chile, migrantes que buscan llevar una forma de vida distinta a la que es habitual en las grandes urbes han impactado el tejido social y cultural de diversas localidades. Muchos de ellos acarrean proyectos utópicos asociados a prácticas creativas, animadas por los sueños de construir una vida mejor en lugares relativamente apartados. En este trabajo tomamos tres iniciativas comunitarias desarrolladas por migrantes y estudiamos las aspiraciones, sueños e intereses que movilizan a los principales agentes y cómo éstos se relacionan afectivamente con el lugar y construyen vínculos con las comunidades locales. Argumentamos que estas intervenciones

\section{Abstract}

The southern Chilean Andes area and its socialcultural sphere have been affected by migrants who aspire to develop new lifestyles different from that of large cities. Encouraged by the dream of achieving a better life in relatively remote places, they bring utopian projects associated with creative practices. This paper analyzes three community-based initiatives developed by migrants, studies their aspirations, dreams and interests and explores how newcomers develop emotional attachment to their new spaces and create new relationships with local communities. It is argued that these interventions give rise to 
conforman "lugares híbridos" caracterizados por una multiplicidad de entendimientos que se expresan en forma simultánea. Concluimos reflexionando sobre la importancia de estudiar lugares construidos con base en entendimientos que transcienden el modo de pensar lineal y material de la modernidad y que anuncian que es posible crear órdenes sociales y espaciales distintos a los que el capital pretende imponer.

PALABRAS CLAVE: MOVILIDAD, UTOPÍA, AFECTOS, LUGARES HÍBRIDOS, CHILE.

Recibido: 01-03-2017.

Aceptado: 31-08-2017.

1 Este artículo fue financiado por la Corporación Nacional de Investigación Científica y Tecnológica (CONICYT), a través del proyecto FONDECYT 1150944 "Espacios discursivos alternos en la Araucanía andino-lacustre: imaginando y creando la identidad del lugar".

2 Chile. Núcleo de Ciencias Sociales - Centro Internacional de Estudios de La Patagonia, Universidad de La Frontera. Correo electrónico: viviana.huilinir@ufrontera.cl.

3 Chile. Departamento de Ciencias Sociales. Núcleo de Ciencias Sociales - Centro Internacional de Estudios de La Patagonia, Universidad de La Frontera. Correo electrónico: hugo.zunino@ ufrontera.cl.

142 revista invi 32(91): 141-160, november 2017 the emergence of "hybrid places" characterized by the generation of diversified simultaneous understandings. It concludes with a discussion about the importance of studying developed spaces created on the basis of understandings that transcend modern linear and material thinking, suggesting the possibility of creating new social and spatial dimensions different than those imposed by capital.

\section{KEYWORDS: MOBILITY, UTOPIA, EMOTIONS, HYBRID PLACES, CHILE.}

Received: 01-03-2017.

Accepted: 31-08-2017.

1 This paper was funded by the National Corporation for Scientific and Technology Research (CONICYT) through FONDECYT Project 1150944 entitled "Alternate Discursive Spaces in the Andean-Lake Region of Araucanía: Imagining and Creating the Identity of the Local Space."

2 Chile.Social Sciences Research Group-Patagonia International Research Center, Universidad de La Frontera. Email: viviana. huilinir@ufrontera.cl.

3 Chile. Department of Social Sciences, Social Sciences Research Group - Patagonia International Research Center, Universidad de La Frontera. Email: hugo.zunino@ufrontera.cl.

ARTICLE: / Mobillty, utopias and hybrid places in the Southern Chilean Andes/Viviana Huiliñir-Curío y Hugo Marcelo Zunino 


\section{Introducción}

En la cordillera de Los Andes del sur de Chile, migrantes nacionales y extranjeros, informados por cosmovisiones indígenas mesoamericanas y avalados por una variedad de discursos biocéntricos, desarrollan proyectos de vida que buscan cristalizar utopías que anuncian un mundo distinto. Este elemento nos alerta sobre nuevas formas de ocupación y uso del territorio que se escapan de lo que es usual en el mundo capitalista contemporáneo y que invitan a pensar en las posibilidades abiertas de transformación social (Espinoza, Zebryte y Zunino, 2014; Zunino, Hidalgo y Zebryte, 2013). Nos referimos a una serie de proyectos alternativos educacionales, prácticas agroecológicas, turismo sustentable y prácticas rituales asociadas a cosmovisiones no modernas que cobran vida en el sur de Chile y articulan discursos contra-hegemónicos apelando a la reconstrucción del sujeto y del lugar (Zunino, Espinoza y Vallejos-Romero, 2016; Zunino y Huiliñir-Curío, 2016 y 2017). El impulso de individuos por recomponer las bases de su existencia terrenal en lugares relativamente apartados respecto de los centros urbanos que comandan el sistema económico mundial y/o regional ha sido estudiado desde la perspectiva de la migración por estilo de vida, la cual gira en torno a la preocupación de conocer las motivaciones de estos sujetos móviles y cómo los lugares receptores se transforman y dan cabida a procesos de desarrollo y re-territorialización con implicancias sociales y culturales diversas (Benson, 2011; Benson y O'Reilly, 2009).

En este trabajo ampliamos el alcance de la literatura sobre migración por estilo de vida, considerando las dimensiones utópicas de esta forma de movilidad contemporánea y el rol que le cabe a las emociones y a las creencias espirituales-religiosas en la formación de lugares que acogen prácticas que, aparentemente, rompen con la homogeneidad que el capital pretende imponer en el espacio. Para ello revisamos la experiencia de los participantes y líderes de tres iniciativas utópicas que, estando interrelacionadas, difieren entre sí en cuanto a sus objetivos y expresiones externas. Los casos de estudio seleccionados son impulsados por migrantes de clase acomodada que en los últimos 15 años se han asentado en distintos lugares de las comunas de Pucón y Curarrehue, en el sur de Chile. Este territorio montañoso y fronterizo está habitado por comunidades mapuches y habitantes locales chilenos, los cuales conforman el basamento social y cultural en donde se insertan estos colonos contemporáneos. Se produce así un lugar de encuentro y disputa, un lugar diverso donde prima la diferencia y que simboliza el impulso existente por construir un mundo distinto e imaginar un futuro mejor. Estamos particularmente interesados en conocer las motivaciones de estos sujetos-migrantes, saber cómo se relacionan con los habitantes locales y reflexionar sobre las implicancias de estas formas 
de ocupación en los procesos de conformación territorial en el sur de Chile.

Este trabajo se estructura de la siguiente manera: en la sección posterior ampliamos los límites de la literatura de migración por estilo de vida, trabajando la noción de utopía y explorando el rol de los afectos en los procesos de constitución del lugar. Luego de describir nuestro caso de estudio y la estrategia metodológica empleada, en la cuarta sección presentamos y discutimos los principales resultados, enfatizando los elementos de bienestar y espiritualidad que se manifiestan en el proceso de construcción comunitaria y los encuentros y aperturas que se dan entre los participantes de las iniciativas examinadas y los habitantes locales más antiguos. Concluimos reflexionando sobre el despliegue de emociones en la constitución de proyectos de vida utópicos y la coexistencia con otras formas de entendimientos, con base en las complejas relaciones entre personas, objetos y lugares.

\section{Migración por estilo de vida y utopía}

El rápido crecimiento poblacional de las últimas décadas en las principales comunas turísticas del sur de Chile se ha asociado a la migración de individuos que buscan desarrollar un estilo de vida más placentero, satisfactorio y significativo en lugares apartados respecto a su lugar de residencia habitual (Zunino, Matossian e Hidalgo, 2012). La literatura sobre esta migración por estilo de vida ha puesto el acento en los sueños y aspiraciones de los sujetos interesados en desarrollar una "nueva vida" en lugares que reúnen condiciones espaciales para realizar cambios en las pautas habituales de comportamiento (Benson, 2011; Benson y O'Reilly, 2009 y 2015), en los impactos que esta forma de movilidad contemporánea tiene sobre las condiciones materiales y las relaciones sociales de los lugares de acogida (Huete, Mantecón y Estévez, 2013; Spalding, 2013) y en las transformaciones sobre el sí mismo que se producen a partir de la constante búsqueda de identificación y descubrimiento personal (Corcoran, 2002). De manera similar a lo que ha sido reportado en la literatura internacional, los migrantes hacia el sur de Chile son ciudadanos con formación universitaria y pertenecientes a sectores acomodados, quienes eligen sitios dotados de amenidades naturales como lugar de destino, privilegiando un estilo de vida al aire libre en contacto con la naturaleza, y buscan distanciarse de los modos de vida de las grandes metrópolis (Zunino e Hidalgo, 2010).

Estudios específicos realizados en Chile sobre comunidades de migrantes por estilo de vida se han relacionado también con intentos deliberados por concretar proyectos de vida no convencionales y que muchas veces apelan a entendimientos que se 
contraponen a lo que es usual en nuestra sociedad contemporánea (Zunino et al., 2013; Zunino et al., 2016). Desde esta perspectiva, el quiebre espacial, social y cultural que propone la realización del proyecto utópico está asociado con el distanciamiento que se produce con respecto al mundo moderno, entendiendo por tal aquel construido por un modo de pensar material e instrumental construido a partir del renacimiento europeo (Mathews, 2006). Para Eisenstadt (2000), el programa cultural y político de la modernidad implicó la separación entre el sujeto que piensa y el mundo natural que lo rodea, y cambios en la concepción de la agencia humana. En efecto, el proyecto conlleva una serie de posibilidades realizables a través de la razón humana, depositando toda la confianza en el desarrollo científico y tecnológico a partir de una agencia humana autónoma. También lleva asociado el dominio de la naturaleza, incluyendo la naturaleza humana. Este proyecto de modernidad implicó un énfasis muy fuerte en la participación autónoma de los miembros de la sociedad en la constitución del orden social y en el acceso de todos los miembros de la sociedad a estos saberes. Es en este contexto que planteamos la existencia de 'utopías modernas', fundadas en la razón moderna, y 'utopías posmodernas', que van más allá y que trascienden la razón moderna fundada en un entendimiento lineal, abstracto y casuístico del mundo que nos rodea (Zunino y Huiliñir-Curío, 2016 y 2017).
Las utopías posmodernas, en particular, avanzan en sus intentos por transformar la realidad social e individual a partir de acciones basadas en entendimientos que se contraponen al conocimiento material y lineal que nos impone la razón instrumental moderna. Para el caso de Chile se ha reportado la emergencia de proyectos comunitarios basados en cosmovisiones meso y norteamericanas, iniciativas de comercio justo con base en el trueque y los saberes locales, y proyectos de desarrollo social e individual basados en entendimientos eco-céntricos (Zunino y Huiliñir-Curío, 2016 y 2017). Parte de los migrantes que han construido su hogar en el sur de Chile en los últimos años pueden ser entendidos como portadores de utopía, representando un síntoma de la creciente crisis que afecta a la sociedad moderna y sugiriendo que las posibilidades de transformación están abiertas y se están manifestando materialmente en la forma de iniciativas que anteponen valores afectivos como el bienestar, el amor, la espiritualidad y la esperanza (Zunino y Huiliñir-Curío, 2016 y 2017).

No obstante estos atributos progresistas y alentadores de la construcción utópica, los intentos de transformación social también deben ser ponderados a la luz de las dificultades y contradicciones que cruzan los esfuerzos por construir una sociedad mejor y más justa. La literatura contemporánea nos advierte que la manifestación de una utopía está vinculada a un acto autoritario al 
establecer un cierre respecto a lo que existe "allí afuera". Esta es la razón por la que la confrontación entre la utopía y el autoritarismo constituye una cuestión política central (Facuse, 2010; Harvey, 2003). Por su parte, Sargisson (2007) advierte que pese a que esta separación es necesaria para el surgimiento creativo de las utopías, el cierre que establece es el elemento más problemático en las comunidades utópicas, dado que la experimentación y el confinamiento espacial pueden favorecer la alienación colectiva y la paulatina degeneración de los proyectos utópicos. Por esta razón, las utopías también pueden relacionarse con espacios peligrosos u oscuras distopías que protegen comportamientos extremos y relaciones de subordinación que pueden emerger a partir de la acción de líderes carismáticos.

En este trabajo asumimos la construcción de lugares utópicos como un proceso situado histórica y espacialmente. Por ello, tomamos tres casos de estudios para comprender la forma en que se articulan las personas y los lugares mediante experiencias que tienen como fundamento prácticas que van más allá de la razón instrumental de la modernidad. Particularmente, en la siguiente sección revisamos cómo la migración utópica se entrecruza con el lugar por medio de los afectos y emociones implícitos en la movilidad y que influyen en la formación de las utopías. Esto nos permitirá capturar los elementos conceptuales necesarios para dirigir nuestro análisis al examen de los tres casos de estudio seleccionados.

\section{Migración, lugar y afecto}

La migración utópica, como señalamos en el apartado anterior, se alimenta de valores afectivos derivados de la búsqueda de lugares en donde puedan concretarse los sueños y esperanzas de un futuro mejor. Esta forma de movilidad voluntaria está, por tanto, intrínsecamente ligada a la noción de 'emoción'. En efecto, no es coincidencia que la palabra emoción, del latín emotio, derive del verbo emovere, que significa "retirar, desplazar de un sitio, mover", y se relacione con el cambio que genera una emoción sobre un estado. Para Wright (2012), las emociones tienen un rol importante en la creación y las formas de desarrollo de personas y lugares, pero han sido evitadas por el pensamiento moderno y su tendencia a equiparar racionalidad con un estado de 'no emoción', en la búsqueda de una pretendida objetividad y neutralidad.

El estudio de las emociones cada día adquiere mayor interés en las ciencias sociales, sobre todo en geografía. Según Bosco (2007), un amplio debate se ha desarrollado en torno a la distinción entre emociones y afectos. De acuerdo a Puente (2012), la idea de afectividad ha reemplazado la 
clásica noción de emoción, debido a los vínculos intersubjetivos que se crean mediante las relaciones afectivas, ya sea entre nosotros, los otros y el entorno. Alejado de todo determinismo, el interés por los afectos radica en la influencia del entorno en la vida emocional de las personas, debido a que existen rasgos del entorno que activan ciertas emociones y sentimientos (Gesler, 1992; Thrift, 1999). De esta manera, la mayor parte de los estudios realizados a la fecha exploran el lazo afectivo entre las personas y los ambientes y en la ubicación de la emoción tanto en los cuerpos como en los lugares (Bondi, 2005; Thien, 2005; Bosco, 2007). Y ha sido precisamente el lugar el concepto geográfico más desarrollado, por capturar los valores contenidos en la experiencia espacial cotidiana.

Los lugares se construyen a partir de marcos de referencia cognitivos, morales y estéticos que informan las concepciones y formas de vincularse con el espacio, según las funciones, fines, valores, relaciones y emociones atribuidos a éste. Los lugares pueden ser cualquier espacio físico reconocido como instancia de relaciones sociales duraderas, tienen una historia socialmente compartida y existen en virtud de concepciones míticas o fundacionales -que explican su origen o desarrollo-, que son afirmadas por los actores sociales (Augé, 2000). La imaginación es uno de los soportes básicos para crear identidad en un lugar, en el sentido que los sujetos se relacionan emocionalmente con los lugares, asignándoles un significado particular que encarna sus historias de vida, ideas, sueños y esperanzas (Gupta y Ferguson, 1997).

Imaginar nuevos mundos ha dado forma a la constitución de 'lugares de esperanza', en donde seres humanos imaginativos articulan iniciativas que buscan recrear una vida y un sentido del "bienestar" diferentes a los predominantes y que simbolizan las opciones abiertas para construir una sociedad distinta por medio de prácticas basadas en el lugar (Escobar, 2004). La esperanza, pulsión que nos ancla al mundo sosteniéndonos frente a la desesperación (Zournazi, 2003), alimenta nuestra capacidad creativa e imaginativa ante los miedos y los anhelos de transformación social. La esperanza como impulso utópico, al igual que el deseo (Levitas, 1990), es un proyecto en curso, un viaje. Al mismo tiempo, es un esfuerzo de voluntad, una estrategia política y un paso importante en la imaginación (Wright, 2008), la cual es crucial para la construcción de utopías.

Tomando como caso de estudio la experiencia de tres iniciativas utópicas emplazadas en Los Andes del sur de La Araucanía, en Chile, revisaremos las aspiraciones, sueños e intereses que movilizan a los migrantes utópicos y examinaremos los fundamentos de los principales agentes de estas iniciativas. También exploraremos los modos como estas iniciativas se materializan y dialogan en el lugar y las relaciones afectivas construidas con el entorno. 


\section{Área de estudio y metodología}

El área turística cordillerana de la región de La Araucanía, conformada por las comunas de Villarrica, Pucón y Curarrehue, es el escenario de un proceso migratorio relevante desde el punto de vista cuantitativo y cualitativo. Si bien no existen levantamientos estadísticos que den cuenta de la magnitud del fenómeno, la evidencia sugiere un fuerte impacto de la migración en el periodo 19922002, pasando la población total de la Comuna de Pucón desde 14.000 a 22.000 habitantes (Zunino e Hidalgo, 2010; Zunino et al., 2012). En términos cualitativos, el impacto de la población migrante sobre el tejido socio-cultural de la localidad, que tradicionalmente se constituía por población chilena y mapuche, ha contribuido a diversificar la vida social y cultural (Zunino et al., 2016) con implicancias en la conformación de una nueva ruralidad chilena (Marchant y Jara, 2017). Este proceso de transformación social se desenvuelve en paralelo con la consolidación de la industria del turismo y con la acción de agentes de gestión público-privada, que convierten a la localidad de Pucón en un "ciudad-fallida" ideada para los turistas y que expresa formas tempranas de segregación socio-residencial (Zunino e Hidalgo, 2010). Del mismo modo, proyectos de carácter extractivo, impulsados por el movimiento de capital nacional y extranjero, se hacen cada vez más presentes en estas comunas, como sucede con numerosas centrales hidroeléctricas de pasada, proyectadas en distintos puntos de Pucón y Curarrehue.

Con el objetivo de explorar la influencia de las emociones en el uso y apropiación del espacio de migrantes utópicos que desarrollan iniciativas de transformación en La Araucanía andino-lacustre, esta investigación considera la experiencia de tres iniciativas utópicas impulsadas por migrantes por estilo de vida (tabla 1), presentes en las localidades de Pucón y Curarrehue: a) La Comunidad de La Montaña, b) La Comunidad para La Vida y c) Rutas Patrimoniales. Las iniciativas se clasificaron en función del tipo de contenido de cada propuesta y los objetivos que persiguen.

La Comunidad de La Montaña, situada en el sureste de la comuna de Curarrehue, es un centro de desarrollo integral donde se organizan eventos y talleres de formación y capacitación, y cuenta con 300 hectáreas, predio que fue comprado el año 1994. Como organización tiene 35 años de antigüedad, tiempo que le ha permitido nutrirse de numerosas corrientes y filosofías 'no occidentales' provenientes de la India y Latinoamérica. También desarrolla actividades de reciclaje y parte de sus lineamientos de acción incluyen el trabajar la autosustentabilidad para la creación de una sociedad con una cultura integral. 


\section{TABLA 1. PERFILES DE LAS INICIATIVAS UTÓPICAS LOCALIZADAS EN LAS COMUNAS DE CURARREHUE Y PUCÓN.}

\begin{tabular}{lll}
\hline Proyecto & Tipo de iniciativa & Tipo de migrantes \\
\hline Comunidad para La Vida & Ecológico-espiritual & Nacionales e internacionales \\
\hline Comunidad de La Montaña & Ecológico-espiritual-desarrollo personal & \\
\hline Rutas Patrimoniales & Desarrollo local y turismo comunitario & \\
\hline
\end{tabular}

Fuente: Elaboración propia, 2017.

La Comunidad para La Vida se inicia el año 2007 con la compra colectiva de 126 hectáreas en un valle cordillerano a 80 kilómetros al este de la ciudad de Pucón. Los propietarios tienen el interés común de desarrollar un proyecto comunitario siguiendo los principios de la organización "Fuego Sagrado", que promueve una cosmovisión fundamentada en un entendimiento de la naturaleza y sus elementos conocida como Camino Rojo ${ }^{4}$. Si bien no opera como un centro de desarrollo, durante la época estival cerca de 200 personas se reúnen a compartir y participar de diversos talleres de sanación, meditación y búsquedas de visión.

Rutas Patrimoniales es un proyecto que tiene su origen el año 2012 y consiste en una red de circuitos turísticos guiados por familias mapuches del valle

4 Movimiento espiritual que recoge distintos elementos discursivos y performativos de los valores tradicionales nativo-americanos y está presente en distintos lugares de América y Europa. del río Trancura, en Curarrehue, pero gestionados por un pequeño grupo de migrantes utópicos, que con apoyo de la Red de Reservas de la Biósfera, de la Unesco, lograron levantar el proyecto, a través del cual invitan a turistas nacionales e internacionales a recorrer y vivenciar los paisajes locales. De esta manera se pretende acercar al turista a la cultura mapuche mediante la gastronomía local y la estadía en distintos puntos de interés de las familias que participan del proyecto. Si bien, en términos formales, esta iniciativa no se gesta desde una comunidad, posee elementos de cooperativismo en su funcionamiento.

Metodológicamente se trata de una investigación de tipo cualitativo-etnográfico que contempló dos etapas: una primera fase de trabajo destinada 
a recabar información secundaria por medio de análisis documental que incluyó los perfiles de las iniciativas disponibles en redes sociales y sitios de internet. Una segunda fase de terreno que se desarrolla desde el año 2015 hasta diciembre de 2016, en la cual se recogieron datos primarios a partir de observación participante y la aplicación de 14 entrevistas en profundidad a diferentes miembros de cada iniciativa. La edad promedio de estas personas es de 45 años y se presenta una distribución equitativa entre mujeres y hombres. El tipo de muestreo fue no probabilístico e intencionado, por lo tanto, la selección de cada participante se realizó por medio de la colaboración de informantes claves. Los criterios de selección fueron: persona migrante (nacional e internacional) mayor de 18 años, con un periodo de residencia en la zona superior a los 6 meses y que participara en alguna de las tres iniciativas.

Las entrevistas fueron grabadas y transcritas para posteriormente ser sometidas a un análisis de contenido simple a través del programa ATLAS Ti. La información fue organizada, codificada y categorizada mediante un análisis abierto inicial y un análisis axial, que permitieron la construcción de categorías temáticas o 'familias', en función de criterios de pertinencia y relevancia. Las categorías se triangularon con el marco teórico que orienta la investigación, a modo de contrastar y relacionar conceptos conducentes a la interpretación de la información construida. Por último, dentro de las consideraciones éticas del estudio se solicitó un consentimiento informado previo a la aplicación de cada entrevista voluntaria. A través de este consentimiento se aseguró el resguardo de la identidad de los participantes, cuyos nombres reales fueron reemplazados por un nombre ficticio, lo mismo que el nombre de cada proyecto.

\section{Nuevas utopías en Los Andes del sur de Chile}

Las iniciativas utópicas emergen cuando miembros y líderes deciden asentarse de forma definitiva en las inmediaciones de Pucón y Curarrehue para concretar un nuevo proyecto de vida en sitios valorados por sus atributos naturales, con rasgos rurales y baja densidad poblacional. Este cambio residencial, empujado por el agotamiento generado por los ritmos de vida en la ciudad y sus formas de movilidad cotidiana, es un aspecto valorado positivamente por los migrantes, quienes en sus testimonios asocian lo 'urbano' a una etapa pasada indeseada. Expresiones como 'auto-exilio' y '(des)arraigo', empleadas por estos migrantes en los relatos acerca de los motivos que empujaron la elección consciente de cambio hacia una vida más satisfactoria, se entremezclan con la idea de 'echar 
raíces' en el nuevo lugar que escogieron para enraizar sus sueños y esperanzas. Este desapego con la ciudad no solo conllevaba un abandono del espacio urbano como el escenario donde se despliega la vida cotidiana. También supone la redefinición de modos de vida personales, sustentada en el reclamo de 'volver a la tierra' como vía para la reinvención individual y la constitución de una vida social más solidaria y consciente en el lugar, facilitado por el contacto permanente con la naturaleza y el acceso a la tierra.

"Al principio me vi así: me arranqué de la ciudad pero me llevé la ciudad para allá. Y pensaba en armar un hostal por aquí, cabañas por allá y los caballos a la montaña, y esto y lo otro. $Y$ ya estaba montando una empresa de turismo ecológico, que estaba bien, pero al final era más de lo mismo en otro formato. Formato outdoor" (hombre, Comunidad para La Vida, octubre de 2015).

La idea de comunidad es una categoría muy presente en las impresiones de los migrantes utópicos, señalada como lo opuesto a 'lo urbano', siendo una instancia de esperanza para el desarrollo de una vida más solidaria. La posibilidad de construir una comunidad fue una motivación significativa para migrar a Pucón y Curarrehue, aspecto mencionado por la totalidad de los participantes. También fue determinante para continuar viviendo en la zona por más tiempo. En efecto, la vida en comunidad es narrada como una vía para cultivar el sentido de pertenencia con el lugar y un acelerador para la reconstrucción personal, a través de la conformación de una segunda familia con personas de mentalidad similar con quienes compartir espacios comunes y de colaboración, aunque comprometieran inversiones económicas y de tiempo para materializarlos. Además, destacan la importancia de la unión, la confianza y el contacto permanente entre las personas del grupo para la concretización de las aspiraciones compartidas.

De manera simultánea, el arraigo al lugar de acogida y su resignificación por medio de la apropiación simbólica del lugar son expresados con más claridad en las iniciativas de carácter espiritual. Desde las narraciones de integrantes de dichas comunidades es posible identificar elementos asociados a las cosmovisiones y corrientes de pensamiento que las sostienen y que son traspasados al lugar por medio de la asignación de nombres y roles a sitios específicos del entorno. Es a partir de cada lugar significativo para las comunidades que se dejan entrever filiaciones afectivas y emotivas con el entorno, promotor de sentimientos amorosos y de resguardo, con base en las vivencias personales de cada sujeto, influidas por los marcos de entendimiento promocionados por la comunidad. Así, la constitución del lugar a partir de la vida en comunidad brinda un sentido social al espacio, mientras que las prácticas que se promueven en las comunidades lo dotan de un sentido ritual. En el caso 
de la Comunidad para la Vida, que persigue la tradición del Camino Rojo, la naturaleza y sus elementos son sagrados, a los que se puede acceder a través de una relación cuidadosa y respetuosa mediante el respeto hacia cuatro elementos básicos: el agua, el fuego, el aire y la tierra, principales símbolos que marcan las pautas de relación de las personas con el mundo visible e invisible, rasgo común de las cosmovisiones no modernas:

"A través de las prácticas rituales, lo que nosotros podemos hacer es el resguardo de este tipo de espacios, para preservar ese espacio en el cual la persona puede permitirse una conexión con algo más grande. Esa conexión, esa impresión, sucede en espacios resguardados (...) al vivir en comunidad, lo que nos permite nutrirnos para prolongar esa conexión en el tiempo y convivirla en todos los espacios en los cuales nos relacionamos" (hombre, Comunidad para La Vida, septiembre de 2016).

\section{Bienestar y espiritualidad}

Las iniciativas utópicas impulsadas por los migrantes por estilo de vida se fundamentan en dos nociones que guardan relación con las características geográficas de Pucón y Curarrehue: bienestar y espiritualidad. La idea de bienestar proviene de la satisfacción y placer de convivir en un entorno natural privilegiado. Montañas, bosques, ríos y cascadas forman parte del deleite que ofrece el lugar, que se entremezcla con los misterios de la experiencia espiritual. Es la materialidad, los valores e imaginarios construidos en torno a un tipo de naturaleza que despierta emociones de paz, tranquilidad y armonía en fusión con ella. La constante alusión a la 'conexión' con elementos del entorno conforma el contenido en el plano discursivo que predomina en los migrantes utópicos, quienes dejan de manifiesto las formas de apego que mantienen hacia otros seres vivos y no vivos.

La montaña, en particular, es un elemento del paisaje trascendente para las vidas de los entrevistados. La verticalidad de las cumbres cordilleranas son valoradas como referencias sagradas, capaces de comunicarse con quien sabe escucharlas y provistas de energía vital:

"El símbolo de la montaña, para diferentes culturas, es el espacio más alto al que uno puede llegar. Como la libertad, la espiritualidad, la conexión con el cielo. Entonces muchos se conectan con el espíritu en sí de la montaña, que tiene que ver con la aspiración de algo mucho mayor (...) También, desde arriba, uno puede ver su vida desde otro espacio, desde otra perspectiva y desde otro lado. Además la montaña es fuerte, posee una energía fuerte, no es una energía suave como el mar, es una energía bien 
firme" (mujer, Comunidad de la Montaña, octubre de 2016).

Para los miembros de la Comunidad para la Vida, la montaña simboliza los poderes de la naturaleza y del Gran Espíritu. Es el lugar de acogida para los buscadores de una visión, que guarda y entrega pautas que orientan a cada buscador. Por lo tanto, representa un espacio pedagógico enfocado en el aprendizaje de principios y valores de lo que ellos llaman "tradición ancestral". La montaña encara al buscador consigo mismo y su fragilidad, recordándole lo inmenso a lo cual está conectado. Es por esta razón que es considerada la "Gran Maestra". La Comunidad de La Montaña también comparte un relato afectivo con la cordillera, porque fue precisamente la figura de una montaña parte de una visión de infancia que recibió el principal líder de esta comunidad, y fue ese sueño el que se concretó con la compra del predio en el cual ahora se materializa su utopía, compartida con los demás integrantes. La señal sobrenatural recibida por el líder de la comunidad posiciona a la montaña como un actor relevante en sus vidas, que ancla la utopía a un lugar específico y le da sentido a la conformación de la comunidad en esta zona.

Del mismo modo afloran otros vínculos afectivos con lugares que obedecen más a una situación específica. Es el caso de Vasco, migrante utópico que participa de la iniciativa de Rutas Patrimoniales. Desde su vivencia, al consultar sobre aquellos elementos significativos del entorno, él se detuvo a reflexionar sobre situaciones de interacción y convivencia con familias mapuches con quienes trabaja por casi cinco años, quienes configuran dichos espacios de aprendizaje y bienestar que suceden en un tiempo y lugar determinados. En su testimonio también se reconoce la indivisible relación entre personas, objetos y lugares con marcada influencia de concepciones de mundo no modernas. La vida social que se desarrolla en los lugares, las materialidades y las experiencias constituyen fragmentos narrativos cargados de contenidos emotivos que evocan la participación de objetos materiales en una situación especial relatada. Estos mecanismos de "personificación de los objetos" (González, 2012) inciden en la creación de relaciones e interacciones recíprocas inseparables que transforman a las "cosas" (naturales y no naturales) en agentes capaces de afectarnos emocionalmente:

“Uno puede llegar molesto a una reunión (...). Y llegas a la conversa ahí, alrededor del fogón, y todo eso se disipa. Digamos, el fuego se lleva toda esta mala energía que en este momento fluye y en esa conversa va generándose un nuevo espacio, un nuevo estado de ánimo. Son todas esas emociones las que a uno le van pasando en Curarrehue. Para mí, ir a las reuniones mensuales no es una obligación, es casi una terapia. Ese espacio está en la conversa con el grupo allá" (hombre, Rutas Patrimoniales, noviembre de 2016). 


\section{Encuentros y aperturas}

Siguiendo con el apartado anterior, los miembros de estas iniciativas se relacionan de forma cercana con familias mapuches del sector. Han sido muchas las oportunidades en que las alianzas comenzaron a forjarse, y por razones diferentes. Un primer contacto ocurre durante las propias actividades que contemplaban las iniciativas utópicas como en el caso de las Rutas Patrimoniales, cuyos integrantes trabajan directamente con familias mapuches. Del mismo modo, la Comunidad de La Montaña ha compartido diversos espacios cotidianos, relacionados con el fortalecimiento de las economías locales, que han adquirido fuerza en el transcurso de los últimos años en Pucón y Curarrehue. Cuando los migrantes utópicos narran los contactos con comunidades mapuches, emergen categorías que explícitamente ponen en juego concepciones de mundo opuestas: la 'occidental' y la 'no-occidental'. Estas categorías son empleadas por los migrantes para recalcar diferencias percibidas en los modos de desarrollo alternativos que se despliegan en Pucón y Curarrehue, diferentes a los fomentados por la economía capitalista moderna. Este aspecto nos parece relevante de mencionar, puesto que el impulso creativo de los migrantes utópicos recibe las influencias del contacto con comunidades mapuches de la zona, quienes también reúnen un cuerpo de conocimiento no moderno con raíces espirituales evidentes:
"Lo que más me llamó la atención es su vínculo con la tierra, o con la huerta o el tema de vivir con lo justo y necesario. No está la ambición que tenemos en el mundo occidental de acaparar y acaparar más, sino también con el día a día ir tratando de subsistir" (hombre, Rutas Patrimoniales, noviembre de 2016).

Un segundo contacto se desencadena con motivo de la instalación de proyectos hidroeléctricos planificados en el territorio, como la central Añihuerraqui, aprobada el año 2015. Esto produjo la aparición de movimientos medioambientales mapuches y territoriales que rechazan toda clase de intervención que conlleve daños ecológicos, sociales y culturales. La acción en ambos sectores, preocupados de preservar la naturaleza y la cultura mapuche de la zona, ha promovido la organización de eventos culturales masivos, jornadas de reflexión y la puesta en marcha de diversos canales de difusión que permitan comunicar la defensa de los ríos y sus aguas. Para ello, los migrantes hacen uso del capital cultural y social que portan, los cuales se han favorecido del acceso a nuevas tecnologías tales como internet y dispositivos audiovisuales que les permiten retratar de manera novedosa los posibles impactos negativos de los proyectos extractivos. A ello se suman intervenciones artísticas y de deporte aventura, que han sido ampliadas a otras partes del mundo con apoyo de las redes internacionales de los migrantes utópicos, quienes no pierden de 
vista las alianzas con las comunidades mapuches que defienden el territorio:

"Nuestra relación con ellos se ha ido fortaleciendo. Hacemos muchos paseos con guías locales. Hoy día también hay varios eventos que se realizan como la feria Walüng, el Puescofest, hay otro que se llama Puesco Long Fest - Long Board, que es un festival para los niños que hacen skate y es uno de los más importantes de Chile. Entonces todos los años están creciendo los movimientos, como el movimiento de personas en esta región" (mujer, Comunidad de la Montaña, octubre de 2016).

Las aperturas y contactos con entendimientos no modernos se reflejan en cómo viven, sienten y se relacionan los migrantes utópicos con el entorno y la sociedad. A modo de ejemplo, la Comunidad para La Vida es un espacio pensado desde la conjunción y el aporte sincrético de diversos entendimientos traídos a la comunidad por diferentes personalidades de los pueblos originarios, y continúan siendo sostenidas por estos mismos, o por quienes les representan, en diferentes encuentros masivos desplegados en la comunidad. Este diálogo permanente entre variadas tradiciones amerindias, articuladas por los cuatro elementos, configuran un espacio abierto e integrado para la generación de una experiencia de proximidad con lo que se considera realmente importante: el cultivo de valores y principios, y la comprensión del amor en todas sus manifestaciones.
Sin embargo, existen diferencias entre las iniciativas respecto a las escalas desde las cuales se expenden. En la caso de Comunidad para La Vida, desde las impresiones de sus miembros no existe una intención concreta de transformación de la sociedad. Más bien los alcances de la iniciativa apuntan a un cambio de conciencia individual, facilitado por posibilidades de acceso a la comunidad, la cual está mediada por la red de socios y el capital que dispongan para poder participar de las ceremonias y rituales de sanación. Aun cuando existe cierta insistencia de los participantes en subrayar que la Comunidad para la Vida no se gesta bajo la posibilidad de concretar una "industria del desarrollo personal", reconocen que este espacio tiene un potencial económico basado en las particularidades paisajísticas del lugar y en el incipiente mercado del coaching, que está aflorando en las inmediaciones de Pucón y Curarrehue y que representa una oportunidad económica y de establecimiento permanente en esta zona a futuro. Por otro lado, la separación con lo externo aparece recurrentemente en los relatos de los entrevistados, quienes forman parte de ese mundo externo y mundano, pero de alguna manera buscan desprenderse de él desde un ámbito más "privado".

"Yo creo que la energía está más bien puesta en eso, en construir algo que en verdad es pequeño en torno a esta gran matriz. Y yo creo que el sistema no lo ve mucho tampoco. Entonces está como medio oculto, porque tampoco hay 
mucha manifestación social, como decía antes (...) Está camuflado. Es como una red invisible, diría yo, que se genera en la sociedad y este es el espacio donde se juntan. A empoderarse, a sanar, a querer cambiar cosas y luego ver cómo lo aplico en el mundo real. (...) O sea, en el mundo exterior" (entrevista 5, hombre, octubre de 2015).

\section{Reflexiones finales: lugares híbridos como nuevas formas utópicas de la movilidad}

Los migrantes utópicos que impulsan proyectos de vida en la cordillera del sur de Chile nos invitan a reflexionar sobre la inseparable relación entre personas, lugares y objetos en el despliegue de emociones y afectos. Estos proyectos utópicos coexisten con otros proyectos no modernos, como los relacionados con el pueblo mapuche, habitantes locales de la zona. Los procesos de negociación con el pueblo mapuche y de reconocimiento del 'otro' son más recurrentes en algunas iniciativas que en otras. La Comunidad de La Montaña y el proyecto Rutas Patrimoniales están en constante diálogo con representantes y familias mapuches de Curarrehue. Estas relaciones se han estrechado, formándose alianzas de cooperación y resistencia frente a proyectos de extracción hídrica que han movilizado a muchas personas y organizaciones de la zona en defensa del medioambiente. Esto demuestra que en el caso de estos proyectos utópicos, instancias de aperturas y críticas frente al modelo moderno pueden avanzar en estructuras más amplias de acción.

Si bien la migración de tipo utópica ha sido explorada desde décadas atrás como una utopía anticultural (Halfacree, 2013), en la actualidad los nuevos grupos que migran hacia lugares prístinos y rurales poseen más recursos que facilitan el acceso a la tierra y una mejor preparación para llevar a cabo proyectos no convencionales. Así, las comunidades de migrantes juegan un papel político al establecer alianzas con comunidades indígenas y desafiar el avance de la modernidad a través de la acción colectiva. No obstante, en algunas comunidades encontramos la conformación de espacios cerrados, con menos vínculos con la realidad en donde se inserta el proyecto. Este camino de reclusión en nichos privados puede tener por consecuencia que estas comunidades se sumerjan en un mundo ilusorio, artificial, exclusivo y segregado, nutrido tan solo por aspiraciones personales de bienestar y degenerando en un negocio personal sin conexión con las formas ideales que aparentemente informaban la construcción de la utopía. Por lo anterior, el desarrollo del elemento espiritual-místico es 
central para que las comunidades se diferencien y así su experiencia sea significativa en términos de construcción de alternativas al mundo moderno.

Por otra parte, la convergencia de cosmovisiones, tradiciones y prácticas en relación a los objetos y su materialidad nos conducen a plantear la idea del surgimiento de 'lugares híbridos' que desafían categorías disponibles. Estos lugares invitan a explorar la conformación de espacios alternos a la modernidad, conformados desde iniciativas utópicas construidas con base en el cultivo de los afectos. La modernidad y sus profundas repercusiones en la regulación racional de los vínculos interpersonales entre los seres humanos y la naturaleza han dado paso a nuevas formas de dominación, como son las emociones y los afectos. Así, el control del 'ánimo' o, en otras palabras, la domesticación de 'lo animal' (ánimo y animal comparten la misma raíz etimológica) grafica el impulso moderno de civilizar o "mejorar el comportamiento de las personas" a partir del dominio de nuestros propias emociones. Este dualismo entre 'lo racional' y 'lo emocional' impide comprender aspectos de la vida que son fundamentales para el funcionamiento de la sociedad y la creación de sueños para el futuro en una compleja realidad (Wright, 2012). En el tema que nos ocupa, los tres casos examinados dan cuenta de la construcción de otras geografías que revelan la construcción utópica de lugares fundada en los sentimientos, representando un contrapunto frente a la tendencia homogeneizadora del proyecto racional moderno.

Indagar en los aspectos utópicos de la migración por medio de la conformación del lugar en relación a los afectos brindó pistas necesarias para explorar nuevos imaginarios e ideas compartidas sobre un tipo particular de relación con la naturaleza. Como señala Halfacree (2013), la migración desempeña un importante rol al reunir a personas con perfiles similares en lugares concretos y momentos determinados, cuyas relaciones dan forma a la producción de lugares variados en la vida cotidiana. Profundizar en detalle los encuentros, aperturas y cómo se practican también las diferencias en ellos, será el futuro desafío que esta investigación propone, sin olvidar las múltiples contradicciones que envuelven a estos procesos.

Finalmente, experiencias como las que examinamos en el sur de Chile nos sugieren la existencia de impulsos que desafían los discursos dominantes, promoviendo otras formas de pensar, sentir y habitar el mundo y que no deben estar ausentes en las discusiones académicas contemporáneas. 


\section{Referencias bibliográficas}

Augé, M. (2000). Los no lugares. Espacios del anonimato. Una antropología de la sobremodernidad. Barcelona: Gedisa.

Benson, M. (2011). The British in rural France: Lifestyle migration and the ongoing quest for a better way of life. Manchester: Manchester University Press.

Benson, M., y O'Reilly, K. (2015). From lifestyle migration to lifestyle in migration: Categories, concepts and ways of thinking. Migration Studies, 4(1), 2037. https://doi.org/10.1093/migration/mnv015.

(2009). Migration and the search for a better way of life: a critical exploration of lifestyle migration. The Sociological Review, 57(4), 608-625. https:// doi.org/10.1111/j.1467-954X.2009.01864.x.

Bondi, L. (2005). Making connections and thinking through emotions: between geography and psychotherapy. Transactions of the Institute of British Geographers, 30(4), 433-448. https://doi. org/10.1111/j.1475-5661.2005.00183.x.

Bosco, F. (2007). Emotions that build networks: Geographies of human rights movements in Argentina and beyond. Tijdschrift voor Economische en Sociale Geografie, 98(5), 545-563. https://doi. org/10.1111/j.1467-9663.2007.00425.x.

Corcoran, M. (2002). The process of reinvention of self: the experiences of returning Irish emigrants. Eire
- Ireland, 37(1-2), 175-191. Recuperado de http:// eprints.maynoothuniversity.ie/1209/.

Eisenstadt, S. (2000). Multiple modernities. Daedalus, 129(1), 1-29. https://doi.org/10.2307/20027613.

Escobar, A. (2004). Más allá del Tercer Mundo: Globalidad imperial, colonialidad global y movimientos sociales anti-globalización. Revista Nómadas, 20, 86-101.

Espinoza, L., Zebryte, I. y Zunino, H. (2014). Análisis del discurso de los migrantes de estilo de vida utópicos en la Patagonia andino-lacustre. En A. Borsdorf, R. Sánchez, R. Hidalgo y H. Zunino (Eds.), Los riesgos traen oportunidades. Transformaciones globales en Los Andes sudamericanos (pp. 263-274). Santiago de Chile: Instituto de Geografía UC.

Facuse, M. (2010). La utopía y sus figuras en el imaginario social. Sociológica, 25(72), 201-213.

Gesler, W. (1992). Therapeutic landscapes: Medical issues in light of the new cultural geography. Social Science y Medicine, 34(7), 735-746. https://doi. org/10.1016/0277-9536(92)90360-3.

González, S. (2012). Una mirada antropológica a la estética y personificación de los objetos. El caso del berimbau en la capoeira Angola en Brasil. Desacatos, (40), 127-140.

Gupta, A., y Ferguson, J. (1997). Culture, power, place: ethnography at the end of an era. En A. Gupta y J. Ferguson (Eds.), Culture, power, place: Explorations in critical anthropology (pp. 1-29). Durham: Duke University Press. 
Halfacree, K. (2013). Utopian communities. En The encyclopedia of global human migration (pp. 1-4). Oxford, UK: Blackwell. https://doi. org/10.1002/9781444351071.wbeghm570.

Harvey, D. (2003). Espacios de esperanza. Madrid: AKAL.

Huete, R., Mantecón, A., y Estévez, J. (2013). Challenges in Lifestyle Migration Research: Reflections and Findings about the Spanish Crisis. Mobilities, 8(3), 331-348. https://doi.org/10.1080/17450101.2 013.814236 .

Levitas, R. (1990). Educated Hope: Ernst Bloch on Abstract and Concrete Utopia. Utopian Studies, 1(2), 13-26. Recuperado de http://www.jstor.org/ stable/20718998.

Marchant, C., y Jara, A. (2017). «Me voy a la montaña a vivir otra vida». Motivaciones y expectativas de migrantes de amenidad y la búsqueda del imaginario verde y del «buen vivir» en la Araucanía andina. En R. Sánchez, R. Hidalgo y F. Arenas (Eds.), Re-conociendo las geografías de América Latina y el Caribe (pp. 133-155). Santiago de Chile: Instituto de Geografía UC.

Mathews, F. (2006). Beyond modernity and tradition: a third way for development. Ethics and the Environment, 11(2), 85-113.

Puente, P. (2012). El valor emocional de la experiencia paisajística. Querencias y paisajes afectivos. Cuadernos Geográficos, (51), 270-284.

Sargisson, L. (2007). Strange places: estrangement, utopianism, and intentional communities.
Utopian Studies, 18(3), 393-424. https://doi. org/10.2307/20719884.

Spalding, A. (2013). Lifestyle migration to Bocas del Toro, Panama: Exploring migration strategies and introducing local implications of the search for paradise. International Review of Social Research, 3(1), 67-86. https://doi.org/10.1515/irsr-2013-0005.

Thien, D. (2005). After or beyond feeling? A consideration of affect and emotion in geography. Area, 37(4), 450-454. https://doi. org/10.1111/j.1475-4762.2005.00643a.x.

Thrift, N. (1999). Steps to an ecology of place. En D. Massey, J. Allen y P. Sarre (Eds.), Human Geography Today (pp. 295-324). Cambridge: Polity Press.

Wright, S. (2008). Practising hope: learning from social movement strategies in the Philippines. En R. Pain y S. Smith (Eds.), Fear: Critical geopolitics and everyday life (pp. 223-233). Aldershot: Ashgate.

Wright, S. (2012). Emotional geographies of development. Third World Quarterly, 33(6), 1113-1127. https://doi.org/10.1080/01436597.2012.681500.

Zournazi, M. (2003). Hope: New philosophies for change. New York: Blackwell.

Zunino, H., Matossian, B., e Hidalgo, R. (2012). Poblamiento y desarrollo de enclaves turísticos en la Norpatagonia chileno-argentina: Migración y frontera en un espacio binacional. Revista de Geografía Norte Grande, (53), 137-158. https://doi. org/10.4067/S0718-34022012000300009. 
Zunino, H., Espinoza, L., y Vallejos-Romero, A. (2016). Los migrantes por estilo de vida como agentes de transformación en la Norpatagonia chilena. Revista de Estudios Sociales, (55), 163-176. https://doi. org/10.7440/res55.2016.11.

Zunino, H., e Hidalgo, R. (2010). En busca de la utopía verde: migrantes de amenidad en la comuna de Pucón, IX Región de La Araucanía, Chile. Scripta Nova, Revista Electrónica de Geografía y Ciencias Sociales, 14, $(331,75)$. Recuperado de http://www. ub.edu/geocrit/sn/sn-331/sn-331-75.htm.

Zunino, H., Hidalgo, R., y Zebryte, I. (2013). Utopian lifestyle migrants in Pucón, Chile: Innovating social life and challenging capitalism. En M. Janoschka y H. Haas (Eds.), Contested spatialities, lifestyle migration and residential tourism (pp. 96-107). New York: Routledge.

Zunino, H. y Huiliñir-Curío, V. (2017). Utopías modernas y posmodernas en el sur de Chile: rupturas y continuidades. En R. Sánchez, R. Hidalgo, y F. Arenas (Eds.), Re-conociendo las geografías de América Latina y el Caribe (pp. 157-181). Santiago de Chile: Instituto de Geografía UC.

(2016). La construcción de lugares alternos en la Cordillera de Los Andes del Sur de Chile: utopía y disrupción de la modernidad. En XIV Coloquio Internacional de Geocrítica: Las utopías y la construcción de la sociedad del futuro (pp. 1-17). Barcelona. Recuperado de http://www.ub.edu/ geocrit/xiv_hugozunino.pdf. 